\title{
Analysis of SEIR model with a single control for COVID-19
}

Naga soundarya lakshmi VSV11 and Sabarmathi $A^{*, 2}$

Received: 20 March 2021/ Accepted: 25 May 2021/ Published online: 18 June 2021

(C)Sacred Heart Research Publications 2017

\begin{abstract}
A SEIR mathematical model for COVID - 19 with a single control vaccination was formulated to analysis the flow of state variables. Properties of Pontryagin's maximum principle is verified and found the optimal levels of control variable. Optimal values of S, E, I, R were derived by equilibrium analysis. Numerical simulations were carried out to exhibit the Susceptible, Exposed, Infectious and Recovery class with and without vaccination which directs that when the susceptible is vaccinated the flow of disease will drop eventually.
\end{abstract}

Key words: SEIR model, Control theory, Pontryagin's maximum principle, Equilibrium Analysis, COVID-19

AMS classification: 34L99, 34L30.

\section{Introduction}

Normally organisms such as bacteria and virus are not harmful but some of them cause disease. It is important to follow some control measures to control the infectious diseases. Here we consider vaccination as our control measure. Vaccination develops an immune in our body against the disease which leads us to control the infection of the disease. In India, we have vaccine for measles and for COVID - 19 developed recently.

Nowadays, mathematical model plays a key role in an analysis of spread and control of the infectious diseases. It describes the transmission process of the diseases. Mathematicians use the optimal control theory for infectious diseases to minimize the cost, infectious individuals and maximize the recovery individuals.

Many Mathematicians used the Optimal control theory for infectious diseases.

\footnotetext{
${ }^{1,2}$ Department of Mathematics, Auxilium College, Vellore, TamilNadu, India.

Emails: ${ }^{1}$ san9sak14@gmail.com, ${ }^{*, 2}$ sabarmathi.a@gmail.com
} 
Specifically, Holly Gaff [2] applied the optimal control for the SIR and SEIR models. Tunde Tajudeen Yusuf and Francis Benyah [9] formulated a SIR model with optimal control of vaccination and treatment. A.Huppert [3] analysed the SIR model with a single control strategy as vaccination.Jakia sultana and Chandra N.Podder 4 ] analysed Nipah virus infections by the optimal control theory. Gul Zaman [1] studied the behaviour of control strategies for various infectious diseases. Nita H.Shah [8] formulated a mathematical model for the Human - Bat population with control strategies. Moftah Bakush [6] studied the impact of vaccination on infectious diseases by the optimal control technique. Moussa Barro [7] has introduced an optimal control for a SIR model with time delay. A. Mhlannga [5] formulated and analyzed the two patch model for Ebola virus disease.

The first COVID - 19 case was reported on 7 March 2020 in Tamilnadu, India. Chennai,the captial of the state being worst affected. The COVID - 19 outbreak was reported on 1 April 2020 in Vellore district of Tamilnadu. In this paper, we have used the data of COVID - 19, Vellore district has been collected from the press release of the Vellore Collectorate and formulated a SEIR model for COVID - 19 with a single control, vaccination. We have analysed the model by optimal control theory and attained that the vaccination decrease the flow of the disease in the state variables.

\section{Formulation of Mathematical model and Control set}

The SEIR model for COVID-19 with a single control is given by the following system of ordinary differential equations.

$$
\begin{array}{r}
\frac{d S}{d t}=\omega N(t)-\beta S(t) I(t)-\mu S(t)-v S(t) \\
\frac{d E}{d t}=\beta S(t) I(t)-\mu E(t)-\alpha E(t) \\
\frac{d I}{d t}=\alpha E(t)-\gamma I(t)-\mu I(t)-d I(t) \\
\frac{d R}{d t}=\gamma I(t)-\mu R(t)+v S(t)
\end{array}
$$

where $S(t), E(t), I(t), R(t)$ are the Susceptible, Exposed, Infectious and Recovery state respectively, $N(t)$ - Total population, $\omega$ - Average birth rate, $\mu$ Average death rate, $\beta$ - Transistion infectious rate, $\gamma$ - Recovery rate , $d$ - disease induced death rate, $\alpha$ - exposed rate. We assume the model with single control $v$ vaccination. 
ISSN: 2456-8686, 5(1), 2021:028-037

https://doi.org/10.26524/cm89

The transmission of SEIR model with a single control for COVID-19 is given as

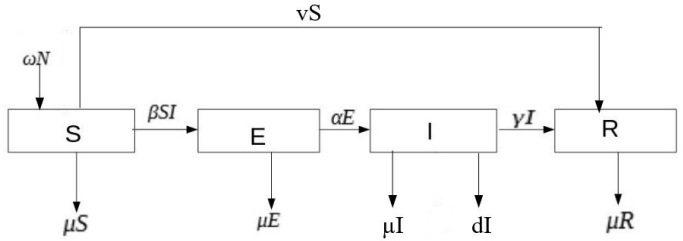

Figure 1: SEIR model with a single control for COVID-19

For this system of equations, we define an objective functional similar as in [6]

$$
J=\min _{v \in U} A I(t)+\frac{1}{2} \int_{0}^{T} B v^{2} d t
$$

which is a free problem system subject to infected.

Where $A I(t)$ is the number of infected individuals, $B$ is the weight parameter.

The control set is defined as

$$
U=\{v: 0 \leq v \leq 1, t \in[0,1]\}
$$

\section{Pontryagin's Maximum Principle}

Pontryagin's maximum principle is a powerful method to compute the optimal value of the control variable. To use this, we have to prove the properties $P_{1}, P_{2}, P_{3}$ and $P_{4}[2]$.

$P_{1}$ : The set of controls and the corresponding state variables is non empty.

$P_{2}$ : The control set $U$ is convex and closed.

$P_{3}$ : The R.H.S. of the state system is bounded by a linear function in the state and control variables.

$P_{4}$ : The integrand of the objective functional is convex on $U$ and is bounded below by $-k_{2}+k_{1}|U|^{\eta}$, with $k_{1}>0, k_{2}>0, \eta>1$.

To prove this we need the following theorem and lemma.

Theorem 3.1 Existence and Uniqueness Theorem: Let $\frac{d y}{d x}=f(x, y), y\left(x_{0}\right)=y_{0}$ and $f(x, y)$ be continuous on a domain $\left\{D=(x, y) / x_{0}-a<x<x_{0}+a ; y_{0}-b<\right.$ $\left.y<y_{0}+b\right\}$ with the Lipschitz condition $\left\|f\left(x, y_{1}\right)-f\left(x, y_{2}\right)\right\| \leq K\left\|y_{1}-y_{2}\right\|$ where $\mathrm{K}$ is a positive integer, then there exist bounded solution in D. Further more, if $f(x, y)$ 
ISSN: 2456-8686, 5(1), 2021:028-037

https://doi.org/10.26524/cm89

is Lipschitz continuous with respect to y on a rectangle $R=\left\{(x, y) / x_{0}-c<x<\right.$ $\left.x_{0}+c ; y_{0}-b<y<y_{0}+b ; c<a\right\}$ then there is a unique solution $y(t)$ in $R$.

Lemma 3.2 If $f(x, y)$ has continuous partial derivative $\frac{\partial f_{i}}{\partial y_{j}}$ on a bounded closed domain $R$, is a set of real numbers. Then it satisfies a Lipschitz condition in $R$.

To prove $P_{1}$ :

From (1),

$$
\begin{gathered}
G_{1}=\omega N-\beta S I-\mu S-v S \\
G_{2}=\beta S I-\mu E-\alpha E \\
G_{3}=\alpha E-\gamma I-\mu I-d I \\
G_{4}=\gamma I-\mu R+v S
\end{gathered}
$$

From (4), $\left|\frac{\partial G_{1}}{\partial S}\right|=\beta I+\mu+v<\infty, \quad\left|\frac{\partial G_{1}}{\partial E}\right|=0<\infty,\left|\frac{\partial G_{1}}{\partial I}\right|=\beta S<\infty, \quad \frac{\partial G_{1}}{\partial R}=0<\infty$ From (5), $\left|\frac{\partial G_{2}}{\partial S}\right|=\beta I<\infty, \quad\left|\frac{\partial G_{2}}{\partial E}\right|=\mu+\alpha<\infty,\left|\frac{\partial G_{2}}{\partial I}\right|=\beta S<\infty, \quad\left|\frac{\partial G_{2}}{\partial R}\right|=0<\infty$ From (6), $\left|\frac{\partial G_{3}}{\partial S}\right|=0<\infty, \quad\left|\frac{\partial G_{3}}{\partial E}\right|=\alpha<\infty,\left|\frac{\partial G_{3}}{\partial I}\right|=\gamma+\mu+d<\infty, \quad \frac{\partial G_{3}}{\partial R}=0<\infty$ From (7), $\left|\frac{\partial G_{4}}{\partial S}\right|=v<\infty, \quad\left|\frac{\partial G_{4}}{\partial E}\right|=0<\infty,\left|\frac{\partial G_{4}}{\partial I}\right|=\gamma<\infty, \quad\left|\frac{\partial G_{4}}{\partial R}\right|=\mu<\infty$ As the partial derivatives are continuous and bounded, it satisfies the Lipschitz condition.

Hence there exist a unique solution of (1).

Thus the set of controls and the corresponding state variables is non empty, $P_{1}$ is satisfied.

To prove $P_{2}$ :

By the definition of $U$, it is closed.

Here $0 \leq \alpha v+(1-\alpha) v \leq 1 \Longrightarrow U$ is convex.

Hence $P_{2}$ is satisfied.

To prove $P_{3}$ :

From (4)-(7),

$$
\begin{array}{r}
G_{1} \leq \omega N-v S \\
G_{2} \leq \beta S I \\
G_{3} \leq \alpha E \\
G_{4} \leq \gamma I+v S
\end{array}
$$


ISSN: 2456-8686, 5(1), 2021:028-037

https://doi.org/10.26524/cm89

The state system can be rewrite in the matrix form,

$$
\bar{G}(t, \bar{X}, U) \leq\left[\begin{array}{cccc}
0 & 0 & 0 & 0 \\
0 & 0 & \beta S & 0 \\
0 & \alpha & 0 & 0 \\
0 & 0 & \gamma & 0
\end{array}\right]\left[\begin{array}{c}
S \\
E \\
I \\
R
\end{array}\right]+\left[\begin{array}{c}
-S \\
0 \\
0 \\
S
\end{array}\right][v(t)]
$$

which can be written as the linear combination of control v,

$$
\begin{array}{r}
|\bar{G}(t, \bar{X}, U)| \leq\|\bar{m}\||\bar{X}|+\|\bar{S}\||v(t)| \\
\leq K_{2}[|\bar{X}|+|v(t)|]
\end{array}
$$

As $\bar{S}$ is bounded, $K_{2}$ is upper bound.

Hence R.H.S. is bounded by the sum of state and control variables, $P_{3}$ is satisfied.

To prove $P_{4}$ :

The control and state variables are non negative and the control variable is also convex and closed.

Then the integrand of the objective functional (2) is convex on the set $U$.

Now,

$$
J=A I(t)+\frac{B v^{2}}{2} \geq-A I(t)+\frac{B v^{2}}{2}=-K_{2}+K_{1} v^{2}
$$

where $K_{1}=\frac{B}{2}$

Here $K_{2}>0$ as it depends on $I, K_{1}>0$ and $\eta=2>1$

Hence $P_{4}$ is satisfied.

Therefore, the control system satisfies the properties of Pontryagin's maximum principle.

\section{Existence of Optimal Control}

As the properties for Pontryagin's maximum principle are satisfied, which converts (1) into a problem of minimizing.

An Hamiltonian $\mathrm{H}$ is defined as 
ISSN: 2456-8686, 5(1), 2021:028-037

https://doi.org/10.26524/cm89

$$
\begin{array}{r}
H(S, E, I, R)=A I(t)+\frac{B}{2} v^{2}+\lambda_{S}(\omega N-\beta S I-\mu S-v S) \\
+\lambda_{E}(\beta S I-\mu E-\alpha E)+\lambda_{I}(\alpha E-\gamma I-\mu I-d I) \\
+\lambda_{R}(\gamma I-\mu R+v S)
\end{array}
$$

and the adjoint system is given by

$$
\begin{array}{r}
\frac{d \lambda_{S}}{d t}=\lambda_{S}(\beta S I+\mu S+v S)-\lambda_{E} \beta I-v \lambda_{R} \\
\frac{d \lambda_{E}}{d t}=\lambda_{E}(\mu+\alpha)-\alpha \lambda_{I} \\
\frac{d \lambda_{I}}{d t}=\lambda_{S} \beta S-\lambda_{E} \beta S+\lambda_{I}\left(\gamma+\mu+d_{-} \gamma \lambda_{R}-A\right. \\
\frac{d \lambda_{R}}{d t}=\mu \lambda_{R}
\end{array}
$$

with the final conditions $\lambda_{S}(T)=\lambda_{E}(T)=\lambda_{I}(T)=\lambda_{R}(T)=0$ for free problem.

Differentiate (8) with respect to $v$,

$$
\begin{array}{r}
{\left[\frac{\partial H}{\partial v}\right]_{v=v *}=0} \\
v^{*}=\frac{S\left(\lambda_{S}-\lambda_{R}\right)}{B}
\end{array}
$$

Hence the optimal control is

$$
v^{*}=\min \left(1, \max \left(0, \frac{S\left(\lambda_{S}-\lambda_{R}\right)}{B}\right)\right)
$$

\section{Equlibrium Analysis}

Now set $s(t)=\frac{S(t)}{N}, e(t)=\frac{E(t)}{N}, i(t)=\frac{I(t)}{N}, r(t)=\frac{R(t)}{N}$ and $r(t)=1-s(t)-$ $e(t)-i(t)$.

$\therefore$ The equation (1) is reduced to the following equations:

$$
\begin{gathered}
\frac{d s}{d t}=\omega-\beta s i-\mu s-v s \\
\frac{d e}{d t}=\beta s i-\mu e-\alpha e
\end{gathered}
$$


ISSN: 2456-8686, 5(1), 2021:028-037

https://doi.org/10.26524/cm89

$$
\frac{d i}{d t}=\alpha e-\gamma i-\mu i-d i
$$

The steady states are $G_{0}(0,0,0), G_{1}(\bar{s}, \bar{e}, 0), G_{2}\left(s^{*}, e^{*}, i^{*}\right)$.

Case(1): Always steady state $G_{0}(0,0,0)$ exists always.

Case (2): For $G_{1}(\bar{s}, \bar{e}, 0)$

Let $\bar{s}, \bar{e}$ be the positive solutions of $\frac{d s}{d t}=0, \frac{d e}{d t}=0$.

From (9) and (10),

$G_{1}(\bar{s}, \bar{e}, 0)=G_{1}\left(\frac{\omega}{\mu+v}, 0,0\right)$, which is the disease free equlibrium.

Case(3): For $G_{2}\left(s^{*}, e^{*}, i^{*}\right)$.

Let $s^{*}, e^{*}, i^{*}$ be the positive solutions of $\frac{d s}{d t}=0, \frac{d e}{d t}=0, \frac{d i}{d t}=0$

From (9),

$$
s^{*}=\frac{\omega}{\left(\mu+\beta i^{*}+v\right)}
$$

From 10,

$$
s^{*}=\frac{(\mu+\alpha) e^{*}}{\beta i^{*}}
$$

From (11),

$$
e^{*}=\frac{(\gamma+\mu+d) i^{*}}{\alpha}
$$

using (14) in (13),

$$
s^{*}=\frac{(\mu+\alpha)(\gamma+\mu+d)}{\alpha \beta}
$$

using $s^{*}$ in 12 ,

$$
i^{*}=\frac{\omega \alpha}{(\mu+\alpha)(\gamma+\mu+d)}-\frac{\mu}{\beta}-\frac{v}{\beta}
$$

From 14),

$$
e^{*}=\frac{\omega}{\mu+\alpha}-\frac{\mu(\gamma+\mu+d)}{\alpha \beta}-\frac{v(\gamma+\mu+d)}{\alpha \beta}
$$

Hence, the endemic equilibrium is

$\left(s^{*}, e^{*}, i^{*}\right)=\left(\frac{(\mu+\alpha)(\gamma+\mu+d)}{\alpha \beta}, \frac{\omega}{\mu+\alpha}-\frac{\mu(\gamma+\mu+d)}{\alpha \beta}-\frac{v(\gamma+\mu+d)}{\alpha \beta}, \frac{\omega \alpha}{(\mu+\alpha)(\gamma+\mu+d)}-\frac{\mu}{\beta}-\frac{v}{\beta}\right)$

\section{Numerical Analysis}

As we found the optimal value of control variable and state variables, we analyse the flow of state variables to study the model with and without vaccination. In Vellore district, the birth rate is $\omega=0.0477$ and death rate is $\mu=0.0203$. The transmission rate of COVID-19 is $\beta=0.011$, the recovery rate is $\gamma=0.96$ and the 
ISSN: 2456-8686, 5(1), 2021:028-037

https://doi.org/10.26524/cm89

death rate is $d=0.017$.

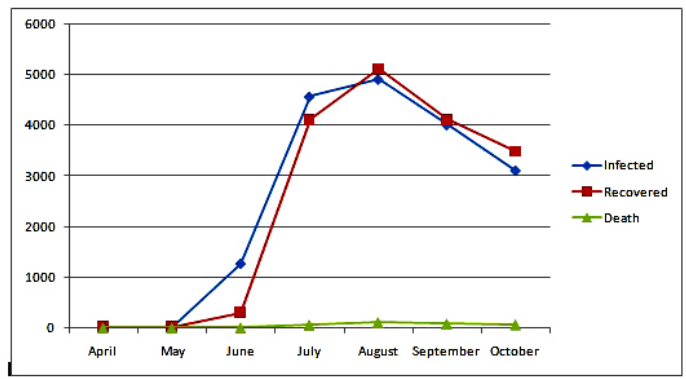

Figure 2: Transmission of COVID-19 in Vellore district

Figure (2) represents the transmission of Confirmed cases, Recovered cases and Death cases of COVID-19 in Vellore district as on 31.10.2020. Figure (3) shows the flow of Susceptible, Exposed, Infectious and Recovery for the model without Vaccination. The flow of disease for the state variables is higher without vaccination.

We study the flow of variable of susceptible. Figure (4) shows that the

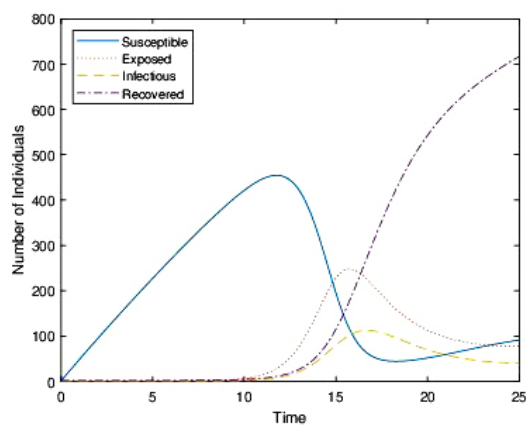

Figure 3: Flow of Variables with respect to Time without vaccination

susceptible with vaccination is less than without vaccination, which shows that vaccination can decrease the spread of the disease. On the study of variable of exposed figure(5) shows that the Exposed with vaccination is reduced from day 1 and without vaccination is higher.

We study the flow of variable of infectious. It is clear from figure (6) that the Infectious with vaccination is is reduced from day 1 while without vaccination is higher. On the study of variable of recovered, figure (7) shows that the recovery with vaccination is higher than without vaccination. Hence the control variable vaccination plays an important role in control the spread of COVID - 19. 


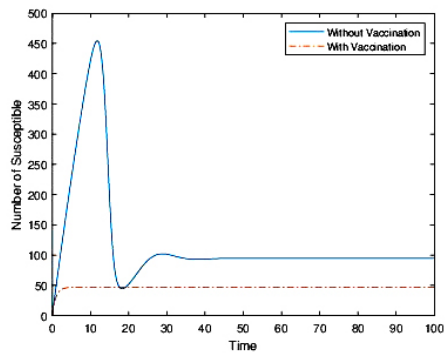

Figure 4: Susceptible class with and without vaccination

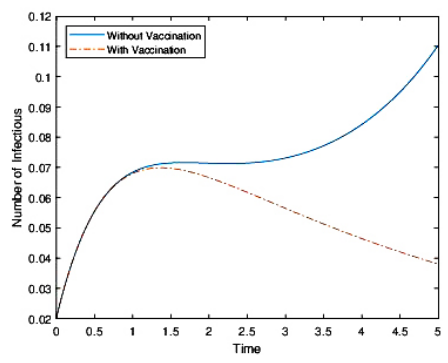

Figure 6: Infectious class with and without vaccination

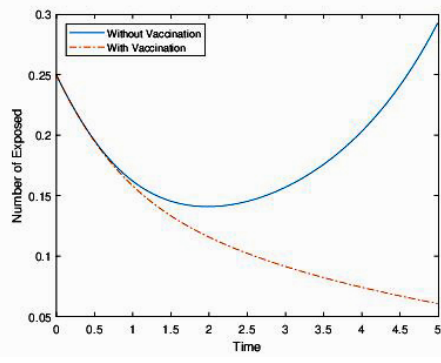

Figure 5: Exposed class with and without vaccination

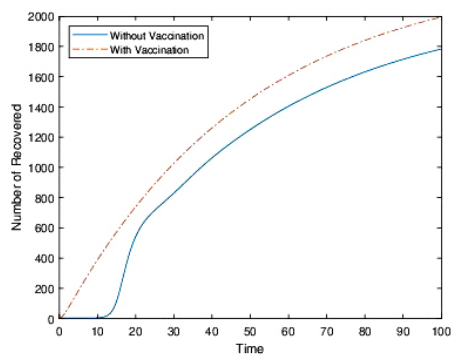

Figure 7: Recovered class with and without vaccination

\section{Conclusion}

A SEIR model with single control measure vaccination is formulated. By Pontryagin's maximum principle and by equlibrium analysis, the optimal levels of controls and variables were analysed. Numerical simulations were given for transition of COVID-19 in Vellore district ,the flow of variables with respect to time without vaccination and the Susceptible, Exposed, Infectious and recovery class with and without vaccination which justifies that the flow of the disease decreases when the susceptible is vaccinated.

\section{References}

[1] Gul Zaman, H.Jung, Delfim F.M.Torres, and Anwar Zeb, Mathematical Modeling and Control of Infectious Diseases,Mathematical Biosciences and Engineering, 6 (3), Hindawi Computational and Mathematical Methods in Medicine , Article ID 7149154, 2017, https://doi.org/10.1155/2017/7149154 
ISSN: 2456-8686, 5(1), 2021:028-037

https://doi.org/10.26524/cm89

[2] Holly Gaff and Elsa Schaefer, Optimal control applied to vaccination and treatment strategies for various epidemiological models, Mathematical Biosciences and Engineering, 6(3), 469-492 (2009).

[3] A. Huppert and G. Katriel, Mathematical modelling and prediction in infectious disease epidemiology,Clinical Microbiology and Infection, 19(11), 999-1005 (2013).

[4] Jakia Sultana and Chandra N. Podder, Mathematical Analysis of Nipah Virus Infections Using Optimal Control Theory, Journal of Applied Mathematics and Physics, 4, 1099-1111 (2016).

[5] A.Mhlanga, Dynamical analysis and control strategies in modelling Ebola virus disease, Advances in Difference Equations , 458 (2019), https://doi.org/10.1186/s13662-019-2392-x

[6] Moftah Bakush, Application of Optimal Control Theory to Infectious Disease, Libyan International Conference on Electrical Engineering and Technologies (LICEET2018), 675-679 (2018).

[7] Moussa Barro, Aboudramane Guiro and Dramane Ouedraogo, Optimal control of a SIR epidemic model with general incidence function and a time delays, CUBO A Mathematical Journal, 20(02), 53-66 (2018).

[8] Nita H. Shah, Niketa D. Trivedi, Foram A. Thakkar and Moksha H. Satia, Control Strategies for Nipah Virus, International Journal of Applied Engineering Research, 13(21), 15149-15163 (2018).

[9] Tunde Tajudeen Yusuf and Francis Benyah, Optimal control of vaccination and treatment for an SIR epidemiological model, World Journal of Modelling and Simulation, 8(3), 194-204 (2012). 